\title{
Resiliencia materna, funcionamiento familiar y discapacidad intelectual de los hijos en un contexto marginado*
}

\author{
Maternal Resilience, Familiar Functioning and Children \\ with Intellectual Disability in a Marginal Context
}

Recibido: junio 14 de 2011 | Revisado: agosto 2 de 2012 | Aceptado: diciembre 23 de 2012

\author{
María del Pilar Roque Hernández ** \\ GuAdAluPe ACle TOMASIN *** \\ Universidad Nacional Autónoma de México, México
}

doi:10.11144/Javeriana.UPSY12-3.rmff

Para citar este artículo: Roque, M. del P. \& Acle, G. (2013). Resiliencia materna, funcionamiento familiar y discapacidad intelectual de los hijos en un contexto marginado. Universitas Psychologica, 12(3), 811-820. doi:10.11144/Javeriana.UPSY12-3. $\mathrm{rmff}$

" Artículo de investigación. Consejo Nacional de Ciencia y Tecnología (CONACyT).

** Facultad de Estudios Superiores Zaragoza, Universidad Nacional Autónoma de México, México. Batalla 5 de Mayo esq. Fuerte de Loreto s. n. Colonia Ejército de Oriente, Delegación Iztapalapa. México, DF. CP 09230. Tel. (+52) 5556230708 ext. 101 y 102. Researcher ID: H-6350-2013. E-mail: roquehmp@gmail.com

**** Researcher ID: H-7535-2013. E-mail: gaclet@unam.mx
RESUMEN

La resiliencia refiere a las interacciones entre el contexto ecológico y la capacidad del individuo para adaptarse positivamente ante la adversidad. Para diseñar e instrumentar la intervención y aportar evidencia a la práctica en educación especial, se analizó la relación entre la resiliencia materna y el funcionamiento familiar, en un contexto marginado. Participaron 76 madres voluntarias, quienes tenían un hijo con discapacidad intelectual inscrito en una escuela de educación especial. Para evaluar resiliencia materna y funcionamiento familiar, se aplicaron dos escalas. Se identificaron relaciones estadísticamente significativas entre las dimensiones de ambos instrumentos, en particular, entre la autodeterminación como característica de resiliencia, con los cinco mediadores personales y ambientales de la misma escala, así como con las dimensiones del funcionamiento familiar.

Palabras clave autores

Educación especial, resiliencia, excepcionalidad.

Palabras clave descriptores

Resiliencia materna, discapacidad intelectual, funcionamiento familiar, autodeterminación, enfoque ecosistémico.

\footnotetext{
A B S T R A C T

The concept of resilience refers to the interactions between the ecological context and the individual's capacity to adapt positively to adversity. We analyzed in a marginal context, the relationship between maternal resilience and family functioning to design and implement the intervention and to provide evidence to practice in special education. 76 volunteer mothers took part in this study. All mothers had a child with intellectual disability in a special education school. Two scales were applied to assess maternal resilience and familiar functioning. Statistically meaningful relationships were identified between the dimensions of both instruments; particularly between self-determination as a characteristic of resilience, with five personal and environmental mediators from the same scale, and with the dimensions of the familiar functioning.

Key words authors

Special education, resilience, exceptionality.

Key words plus

Maternal resilience, intellectual disability, familiar functioning, self-determination, ecosystemic approach.
} 


\section{Introducción}

El estudio relativo a la resiliencia personal surgió a partir de los trabajos con énfasis en el riesgo, realizados desde el enfoque de la psicopatología del desarrollo: las investigaciones de Rutter (1987) y Werner y Smith (1992) han contribuido a evidenciar que una misma adversidad puede originar diferentes resultados, de ahí que los elementos personales y del medio son importantes para explicarlos. A partir de entonces, se ha incrementado el abordaje de este constructo en áreas como la psicología, momento en que las explicaciones acerca de por qué algunas personas logran superar la adversidad, se han elaborado desde diversas perspectivas teóricas que priorizan aspectos individuales y/o su relación con el ambiente o contexto.

Desde el enfoque ecosistémico se parte de que el desarrollo es un proceso continuo de adaptación que no sucede en el vacío, sino que es producto de transacciones continuas entre el individuo y los contextos físicos, sociales y culturales en los cuales se desarrolla (Breakwell, 2009; Henley, 2010; Rodríguez, Alvarado \& Moreno, 2007). Al estudiar la resiliencia, se hace prioritario conocer las interacciones entre el contexto ecológico y la capacidad del sistema dinámico -en este caso de la persona- para adaptarse positivamente (Henley, 2010; Masten, 2007; Obrist, Pfeiffer \& Henley, 2010; Secretaría de Educación Pública [SEP], 2011).

Por tanto, la resiliencia del individuo involucra: 1) sus características, esto es, su comportamiento, lo que hace para influir en los elementos de su o sus ambientes y adaptarse positivamente al evento adverso y 2) el conocimiento de cuáles son y cómo se relacionan los mediadores personales y ambientales que intervienen, al amortiguar (cuando actúan de forma positiva) o agravar (cuando lo hacen de manera negativa) el impacto de una adversidad específica en contextos particulares y favorecer o no las características resilientes (Henley, 2010; Obrist et al., 2010; Roque, Acle \& García, 2009; Waller, 2001).

La presencia de los mediadores origina el carácter multideterminado de la resiliencia, y el hecho de que las personas resilientes sean diferentes unas de otras apunta a la existencia de un constructo dinámico y multidimensional, en el cual el individuo no es visto de manera aislada, ya que las interacciones que mantiene dentro y fuera de la familia lo impactan directamente (Breakwell, 2009; Spratt, 2010). Como Masten (2007) señaló al estudiar la resiliencia desde esta perspectiva, el foco está en un análisis multinivel y en las dinámicas de adaptación y cambio.

En este sentido, la familia es vital para el desarrollo de sus miembros, en especial, cuando existen hijos con discapacidad (Gardynik \& McDonald, 2005; Gargiulo, 2012; Henley, 2010; Rodríguez et al., 2007). La discapacidad se refiere a las limitaciones del individuo para actuar y participar, resultantes de la interacción entre sus propias características y las de la sociedad en la que vive (World Health Organization [WHO], 2011). De acuerdo con la WHO (2011), aproximadamente $10 \%$ de la población mundial, o 650 millones de personas, presentan discapacidad -cifra que ha aumentado 50 millones con relación a 1990-, por lo que se busca mejorar su calidad de vida e inclusión, con esfuerzos globales, regionales y nacionales, entre ellos, lograr la atención adecuada por parte de los padres (Organización de las Naciones Unidas, 2006).

Se ha reportado que lo que afecta positiva o negativamente la vida de un niño con discapacidad, no solo depende de sus características, sino de la forma en que sus padres lo ven, lo tratan y de lo que hacen por él (Gardynik \& McDonald, 2005; Gargiulo, 2012; Obrist et al., 2010). Sin embargo, los estudios realizados con adultos sobre resiliencia se han dirigido más a identificar y promover la resiliencia en los hijos, siendo los padres básicamente un recurso de información sobre estos. No obstante, como Barudy y Dantagnan (2005) indicaron, es importante la parentalidad resiliente, esto es, la capacidad que tienen los padres para proporcionar los cuidados necesarios a los hijos y para ayudarles frente a los sucesos adversos que les toca vivir.

Es bien sabido que los padres de niños con discapacidad están más en riesgo de incrementar el estrés que aquellos que tienen hijos con desarrollo normal. Como anotaron Flippin y Crais (2011), las madres o tutoras de hijos con discapacidad son 
más propensas a problemas relacionados con sus cuidados, como depresión, ansiedad, aislamiento social y baja autoestima. De ahí que se precisa desarrollar investigación que dé cuenta de la resiliencia materna en beneficio del hijo, máxime cuando este presenta alguna excepcionalidad (Gargiulo, 2012), en la que se determinen cuáles son los comportamientos de las madres en beneficio de sus niños y los mediadores que favorecen o no la presencia de dichos comportamientos.

El funcionamiento familiar positivo es un recurso que puede contribuir para que los padres manejen la discapacidad que presenta su hijo (Gargiulo, 2012; Walsh, 2003). El funcionamiento negativo de la familia se asocia con la negación o evitación de lo relacionado con la discapacidad, con una comunicación ineficaz, con la falta de búsqueda de soluciones, con la carencia de fortalezas entre sus miembros y con la falta de involucración de estos para actuar en pro de quien presenta la discapacidad (Waller, 2001); más aún, se le adjudican las carencias del sistema familiar y se utiliza como el "chivo expiatorio" de los problemas (Pedraza-Vargas, Perdomo-Carvajal \& Hernández-Manrique, 2009).

El desarrollo y aplicación de instrumentos psicológicos que sean válidos y confiables, dirigidos a identificar la resiliencia de las personas ante adversidades específicas, contribuye a clarificar el constructo y a desarrollar herramientas prácticas para intervenir y enriquecer la resiliencia individual en ambientes reales. Se precisa, como señaló Luthar (2006), identificar a los padres como una variable dependiente, esto es, conocer cómo las características de los hijos pueden afectar sus conductas y funcionamiento psicológico; lo que cobra mayor importancia en familias de bajos ingresos y de regiones socioeconómicas en desventaja. En este sentido, hay que considerar que las circunstancias de vida que son normativas en un contexto sociocultural dado pueden no serlo en otro, de ahí que los hallazgos sobre resiliencia basados en una población no pueden ser generalizados a otras (Masten, 2007; Waller, 2001): la resiliencia refleja elementos culturales.

En México, existen 5'739.270 personas con algún tipo de discapacidad, lo que representa el $5.1 \%$ de la población total; de este último porcentaje, el $8.5 \%$ presenta discapacidad intelectual, siendo la cuarta discapacidad con más presencia a nivel nacional (Instituto Nacional de Estadística, Geografía e Informática [INEGI], 2010b). Pese a que es sabido que en el país las madres continúan, de forma desproporcionada, a cargo de los menores con discapacidad, se precisa de mayor investigación empírica que, desde el constructo de resiliencia, identifique cómo los afectan discapacidades específicas, qué es lo que hacen por los hijos y con qué fortalezas y recursos cuentan.

En el modelo actual de servicios de educación especial del país (SEP, 2011), basado en el mismo enfoque ecosistémico, se considera que para lograr una respuesta educativa pertinente y mejorar el aprendizaje de los alumnos con discapacidad máxime si habitan en zonas marginadas y asisten a escuelas de educación especial-, se requiere de la participación comprometida sobre todo de las madres, así como el estudio de los contextos, de los procesos que se dan en y entre ellos y de las actitudes y percepciones de los involucrados, por cuanto imponen límites o inducen a determinados comportamientos. A partir de lo señalado, en el presente trabajo se analizó en un contexto urbano marginado de México, la relación entre la resiliencia de madres cuyos hijos presentan discapacidad intelectual y el funcionamiento de sus familias, para diseñar e instrumentar la intervención psicológica y aportar evidencia a la práctica en educación especial.

\section{Método}

\section{Contexto y escenario}

Se trabajó en una escuela pública de educación especial (Centro de Atención Múltiple, CAM), ubicada en Iztacalco (Distrito Federal, México), en una zona urbana marginada, caracterizada por condiciones socioeconómicas precarias, altos índices de delincuencia, drogadicción y asentamientos urbanos irregulares. Iztacalco es la segunda zona industrial más importante de la ciudad y ocupa el noveno lugar entre las delegaciones, por cantidad de 
personas en condiciones de marginalidad (INEGI, 2010a). EL CAM pertenecía al programa de Escuelas de Calidad, era de turno continuo (8:00 a 16:00 hrs), y buscaba atender a la diversidad, para lo cual lograr que los padres realizaran acciones a favor de sus hijos, constituía una premisa importante.

\section{Participantes}

En el estudio participaron 76 madres voluntarias con al menos un hijo diagnosticado con discapacidad intelectual (55.07 \% de la población escolar total), de las cuales el $91 \%$ eran madres biológicas, con 39 años de edad en promedio $(D E=9.701$, Rango $=24-74)$, dos hijos $(D E=1.126$, Rango $=$ 1-7) y bachillerato inconcluso. El $63 \%$ de las madres vivía con su cónyuge y $53 \%$ percibía un salario mensual entre $\$ 500$ y $\$ 1.500^{1}$. La muestra fue no probabilística intencional.

Las características de los hijos de las participantes eran: a) $57 \%$ hombres; b) nueve años de edad en promedio $(D E=2.746$, Rango $=4-14)$; c) diagnóstico de discapacidad intelectual, realizado por institución pública y d) 37 \% contaba trimestralmente con una beca otorgada por el Gobierno (\$700.00).

\section{Herramientas}

La información se obtuvo principalmente a través de los siguientes tres instrumentos:

\section{Escala de Resiliencia Materna} (Roque et al., 2009)

Contiene 45 reactivos con valores propios mayores a 1, que explican el $50.19 \%$ de la varianza total, con un alpha de Cronbach global de 0.919; utiliza una clasificación de cinco puntos tipo Likert (desde nunca hasta siempre), que se acompaña de una escala pictórica. Desde un enfoque ecosistémico, el constructo apunta a la interacción entre las características de la madre (comportamientos) para la aten-

1 En México, en 2009, el salario mínimo diario era de \$54.80. En moneda nacional, un dólar norteamericano equivalía a \$13.27. ción del hijo con discapacidad, con los mediadores que determinan el impacto de dicha discapacidad y favorecen o no la adaptación positiva. La escala está conformada por seis factores: uno para identificar la autodeterminación como característica de resiliencia y cinco mediadores relacionados: tres personales (desesperanza, fe espiritual y rechazar la responsabilidad personal) y dos ambientales (falta de apoyo de la pareja y recursos limitados para satisfacer necesidades).

Escala de Funcionamiento Familiar

(García-Méndez, Rivera, Reyes-

Lagunes \& Díaz-Loving, 2006).

Elaborada desde un enfoque sistémico, contiene 45 reactivos con valores propios mayores a 1 ; explica el $56 \%$ de la varianza total y su consistencia interna global es 0.853. Incluye una clasificación de cinco puntos tipo Likert (desde nunca hasta siempre) y cuatro dimensiones: ambiente familiar positivo, hostilidad/evitación del conflicto, mando/problemas en la expresión de sentimientos y cohesión/ reglas.

Registros de observación y documentación oficial del centro escolar.

\section{Procedimiento}

Se obtuvo el consentimiento informado del personal escolar y supervisión de zona, lo que permitió el acercamiento a las participantes potenciales. La aplicación de instrumentos fue individual y el tiempo promedio para cada escala fue de 30 minutos.

\section{Resultados}

Se calcularon correlaciones de Pearson para analizar primero, la relación entre las dimensiones de cada instrumento, y posteriormente, para identificar la existente entre las de resiliencia materna y del funcionamiento familiar. Los resultados de la relación entre las dimensiones de la resiliencia materna se muestran en la Tabla 1. Se encontraron 
TABLA 1

Correlación entre las dimensiones de resiliencia materna

\begin{tabular}{lccccc}
\hline \multicolumn{1}{c}{ Dimensiones } & Desesperanza & $\begin{array}{c}\text { Falta de apoyo } \\
\text { de la pareja }\end{array}$ & $\begin{array}{c}\text { Recursos limitados para } \\
\text { satisfacer necesidades }\end{array}$ & Fe espiritual & $\begin{array}{c}\text { Autodeter- } \\
\text { minación }\end{array}$ \\
\hline $\begin{array}{l}\text { Desesperanza } \\
\text { Falta de apoyo de la pareja }\end{array}$ & 1 & & & & \\
Recursos limitados para satis- & $0.607^{* *}$ & 1 & & & \\
facer necesidades & $0.694^{* *}$ & $0.48^{* *}$ & 1 & & \\
$\begin{array}{l}\text { Fe espiritual } \\
\text { Autodeterminación }\end{array}$ & $-0.338^{* *}$ & -0.217 & -0.217 & 1 & \\
$\begin{array}{l}\text { Rechazar la responsabilidad } \\
\text { personal }\end{array}$ & $-0.749^{* *}$ & $-0.516^{* *}$ & $-0.621^{* *}$ & $0.491^{* *}$ & 1 \\
\hline
\end{tabular}

$* * p=0.01 ; * p=0.05$.

Fuente: elaboración propia.

TABLA 2

Correlación entre las dimensiones de la escala de funcionamiento familiar

\begin{tabular}{lccc}
\hline \multicolumn{1}{c}{ Dimensiones } & $\begin{array}{c}\text { Ambiente } \\
\text { familiar positivo }\end{array}$ & $\begin{array}{c}\text { Hostilidad/evitación } \\
\text { del conflicto }\end{array}$ & $\begin{array}{c}\text { Mando/problemas en la } \\
\text { expresión de sentimientos }\end{array}$ \\
\hline Ambiente familiar positivo & 1 & & \\
Hostilidad/evitación del conflicto & $-0.732^{* *}$ & 1 & 1 \\
Mando/problemas en la expresión de sentimientos & $-0.727^{* *}$ & $0.834^{* *}$ & $-0.296^{* *}$ \\
Cohesión/reglas & $0.591^{* *}$ & $-0.417^{* *}$ & \\
\hline
\end{tabular}

$* * p=0.01$.

Fuente: elaboración propia.

correlaciones negativas significativas entre la autodeterminación de las madres de hijos con discapacidad intelectual, con: dos mediadores personales (desesperanza y rechazar la responsabilidad personal) y los dos ambientales (recursos limitados para satisfacer necesidades y falta de apoyo de la pareja).

La desesperanza materna, por su parte, correlacionó de forma positiva y significativa, principalmente con: el propio rechazo en cuanto a la responsabilidad para con el hijo con discapacidad; la presencia de recursos limitados para satisfacer las necesidades de este y la falta de apoyo de la pareja para atenderlo. El rechazo de la madre a dicha responsabilidad personal, correlacionó positiva y básicamente con los dos mediadores ambientales ya señalados. Por último, se destaca la relación positiva identificada entre ambos mediadores ambientales.

Respecto al funcionamiento familiar (Tabla 2), se identificó que cada una de las dimensiones positivas de la escala -esto es, el ambiente familiar positivo y cohesión/reglas-, se asoció de forma negativa y signi- ficativa con las dos dimensiones negativas: hostilidad/ evitación del conflicto y mando/problemas en la expresión de sentimientos. Las correlaciones positivas se presentaron entre: mando/problemas en la expresión de sentimientos y hostilidad/evitación del conflicto, y entre ambiente familiar positivo con cohesión/reglas.

Respecto a la relación entre las dimensiones de ambas escalas (Tabla 3), se encontró que la autodeterminación de la madre, se relacionó de forma significativa con las cuatro dimensiones del funcionamiento familiar: de forma positiva principalmente con ambiente familiar positivo y de forma negativa, con mando/problemas en la expresión de sentimientos y hostilidad/evitación del conflicto. Finalmente, cuatro de los mediadores de resiliencia materna -a excepción de la fe espiritual- se asociaron principalmente con tres dimensiones del funcionamiento familiar: negativas con ambiente familiar positivo y positivas, tanto con hostilidad/ evitación del conflicto, como con mando/problemas en la expresión de sentimientos. 
TABLA 3

Correlación entre las dimensiones de resiliencia materna y del funcionamiento familiar

\begin{tabular}{|c|c|c|c|c|c|c|}
\hline \multirow[b]{4}{*}{$\begin{array}{l}\text { Funcionamiento } \\
\text { Familiar }\end{array}$} & \multicolumn{6}{|c|}{ Resiliencia Materna } \\
\hline & \multirow[b]{3}{*}{ 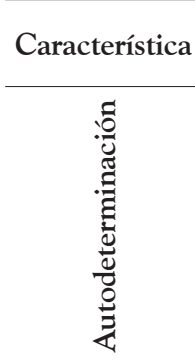 } & \multicolumn{5}{|c|}{ Mediadores } \\
\hline & & \multicolumn{3}{|c|}{ Personales } & \multicolumn{2}{|c|}{ Ambientales } \\
\hline & & 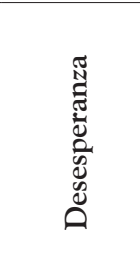 & 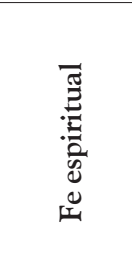 & 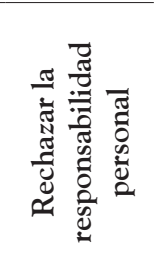 & 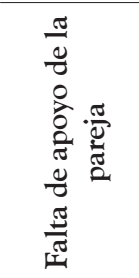 & 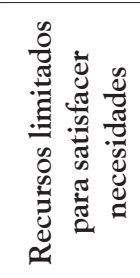 \\
\hline Ambiente familiar positivo & $0.585 * *$ & $-0.534 * *$ & 0.118 & $-0.453 * *$ & $-0.334 * *$ & $-0.565 * *$ \\
\hline Hostilidad/evitación del conflicto & $-0.497^{* *}$ & $0.513 * *$ & -0.142 & $0.464 * *$ & $0.372 * *$ & $0.511^{* *}$ \\
\hline Mando/problemas en la expresión de sentimientos & $-0.51 * *$ & $0.539 * *$ & 0.007 & $0.533^{* *}$ & $0.551^{* *}$ & $0.529 * *$ \\
\hline Cohesión/reglas & $0.287 *$ & -0.203 & 0.001 & -0.12 & $-0.28 *$ & -0.206 \\
\hline
\end{tabular}

$* * p=0.01 ; p=0.05$

Fuente: elaboración propia.

\section{Discusión}

El presente trabajo da cuenta de elementos específicos interrelacionados que se suceden respecto a la resiliencia de madres de hijos con discapacidad intelectual en un contexto particular de riesgo, lo que responde a una necesidad actual de investigación (Flippin \& Crais, 2011; Luthar, 2006; SEP, 2011; Spratt, 2010).

La existencia de múltiples definiciones sobre resiliencia hace que no haya un acuerdo entre los investigadores respecto a su significado y en cuanto a las dimensiones que la conforman -las cuales son diversas y han sido estudiadas en muchas ocasiones de forma independiente y no como parte de este constructo-, e impacta en la presencia de distintas formas para su evaluación e investigación. No obstante, identificar la presencia de la autodeterminación en las madres ante la discapacidad intelectual -como característica de resiliencia y desde un enfoque ecosistémico- es fundamental, pues da cuenta de las acciones que estas pueden realizar en apoyo al desarrollo e inclusión de sus hijos, pese al contexto de vulnerabilidad en el que habitan y a los mediadores personales y ambientales relacionados que pueden agravar el impacto de dicha adversidad.

La autodeterminación referida a las acciones que la madre realiza para atender adecuadamente a su hijo, pese a la discapacidad intelectual que este presenta, correlacionó con los cinco mediadores personales y ambientales que conforman la escala aplicada, así como con el mediador relativo al funcionamiento familiar, los cuales en interacción pueden contribuir a amortiguar el impacto de la discapacidad. Ello apunta a que las madres son agentes activos de información, formación y comportamientos, que se explican al conocer el funcionamiento de sus familias, así como de las conexiones de estas con los elementos de otros sistemas con los que mantienen relaciones (SEP, 2011).

Lo anterior pone de manifiesto que la resiliencia es multideterminada y multicausal, por lo que al estudiarla no se priorizan las características de personalidad del individuo o algún atributo personal subjetivo, sino que es un proceso de desarrollo dinámico, en el que está presente la experiencia de adversidad relevante para la persona y en la cual la presencia e interacción entre los mediadores personales y del ambiente familiar y social en general, pueden contribuir a superar dicha adversidad.

En este sentido, hay que considerar que las circunstancias de vida que son normativas en un contexto sociocultural dado pueden no serlo en otro; de ahí que los hallazgos sobre resiliencia basados en una población y ante una adversidad específica, no pueden ser generalizados a otras (Waller, 2001). 
Como indicó Farkas-Klein (2008), la sensitividad cultural de un instrumento es importante: instrumentos desarrollados en un país pueden incluir tareas o actividades que no sean familiares para los padres de otros países o los reactivos pueden variar en su significado social. De ahí la importancia de diseñar, validar y aplicar otros instrumentos que, como los empleados en el presente estudio, consideren las particularidades socioculturales de los destinatarios y permitan identificar tanto otras características de resiliencia materna como diversos mediadores personales y ambientales relacionados.

En este sentido, y a partir de los resultados de la presente investigación, mientras más autodeterminada sea la madre -lo que refiere a que tenga la habilidad de tomar decisiones por sí misma, se fije metas, actúe para lograrlas, reflexione, evalúe sobre su ejecución y realice los ajustes necesarios a partir de lo logrado para conseguir la atención adecuada del hijo con discapacidad intelectual-, más: a) pensará y sentirá que puede lograr los eventos relacionados con el menor, evaluándolos como deseables e importantes; b) aceptará la responsabilidad que tiene en cuanto a las acciones que realiza o los problemas relativos a su hijo; c) percibirá que cuenta con apoyos internos y externos a su familia -entre ellos los de su pareja-, para satisfacer las necesidades económicas, de salud y educación del menor y d) considerará que la fe espiritual le da paz, tranquilidad y fuerza para criar, sacar adelante y proporcionar la atención necesaria a su hijo con discapacidad.

Estas habilidades -incluidas y relacionadas con la autodeterminación de las madres- coinciden con las reportadas por Breakwell (2009), Henley (2010) y Wehmeyer y Field (2007) acerca del mismo concepto: pensar antes de actuar, percibir diferentes opciones para elegir cuál emplear en una situación particular, actuar activamente y adaptarse positivamente para cambiar el medio. De manera contraria, y ante situaciones estresantes, cuando la madre cree que sus acciones para atender al menor no son eficaces y no está satisfecha con esto, se rendirá más rápidamente, se atribuirá el fracaso y, por tanto, se presentarán más emociones negativas (Farkas-Klein, 2008).
Para Walsh (2003) las características de resiliencia de las personas dependen de los recursos familiares, escolares y comunitarios; sin embargo, los resultados del presente estudio constatan que la autodeterminación de la madre ante la discapacidad intelectual del hijo, resulta de la interacción tanto de tales recursos ambientales como de los propios. Esto coincide con los determinantes señalados por Breakwell (2009), Henley (2010) y Wehmeyer y Field (2007) sobre autodeterminación en general: a) ambientales, como el acceso a la información y la disponibilidad de apoyo social ante la adversidad y b) de la persona misma, entre ellos, la capacidad para aceptar la responsabilidad de sus actos, su confianza para tomar decisiones y realizar acciones, sus patrones de comunicación y la selección y logro de metas.

Es claro que al estudiar la resiliencia materna deben considerarse los mediadores que permiten predecir un resultado ya sea favorable o desfavorable, ya que estos pueden tener un impacto acumulado en la persona. Estudiar los mediadores relacionados con resultados positivos y negativos específicos permite entender la interacción entre estos y su influencia en la resiliencia. La relación identificada entre desesperanza y la falta de apoyo de la pareja, así como con recursos limitados para satisfacer necesidades, coincide con los hallazgos reportados por Gardynik y McDonald (2005) respecto a madres de hijos sobresalientes con problemas para aprender, quienes no contaban con apoyo del cónyuge, mostraban menor bienestar psicológico y responsabilidad hacia el hijo, lo que se exacerbaba ante problemas económicos y de salud física.

El presente trabajo aporta información acerca de la importancia del padre en relación con la resiliencia materna, al identificar que: entre mayor sea la falta de apoyo de este para la atención del hijo con discapacidad, mayor será el rechazo de la madre para asumir la responsabilidad del mismo y menor su autodeterminación. Esta falta de apoyo se identificó relacionada también con el funcionamiento familiar: presencia de un ambiente familiar negativo, hostilidad, problemas para expresar sentimientos y falta de cohesión/reglas. Ello añade información a lo señalado por Flippin y Crais (2011) 
cuando afirman que existe una ausencia de trabajos que reporten la presencia de los padres (hombres) en las acciones de investigación y de intervención temprana de los hijos con discapacidad, pese a que sus estilos de interacción pueden contribuir al desarrollo del lenguaje y del juego simbólico de niños normales y con autismo, lo que beneficia además a sus familias, al reducir la tensión existente entre sus miembros y enriquecer la forma en que padres y madres responden a la adversidad.

En relación con la fe espiritual, Rodríguez et al. (2007) y Whitley (2012) coincidieron al afirmar que esta proporciona a la persona un sentido de alivio y control ante eventos adversos o la discapacidad misma, por lo que puede contribuir a reducir la tensión, la dependencia respecto a los demás e influir positivamente en la conducta y en cómo se enfrenta lo adverso, máxime cuando existen limitaciones económicas. Cuestiones que aunque requieren de posteriores análisis y mayor sustento al aplicarlos a la resiliencia materna ante la discapacidad intelectual, apuntan a los resultados del presente estudio realizado en una comunidad marginada: entre más fe espiritual tenga la mujer, existirá menor desesperanza y rechazo de la responsabilidad en cuanto al hijo, así como mayor autodeterminación. Finalmente, lo reportado por Rodríguez et al. y Whitley da cuenta de que la fe personal impacta prioritariamente en el individuo mismo, lo que apunta a la identificada falta de asociación significativa con el funcionamiento familiar.

En la familia, y máxime cuando existe algún hijo con discapacidad, es claro que tanto esta como sus integrantes experimentan cambios a corto y largo plazo y que dichos cambios requieren de un ajuste, porque afectan tanto a su funcionamiento como a la forma en que los miembros responden a las circunstancias adversas. En este sentido, las familias pueden usar sus fortalezas para reducir de manera efectiva dichos cambios (García-Méndez et al., 2006; Rodríguez et al., 2007). En el presente trabajo, se identificó al funcionamiento familiar como un mediador importante relacionado con la resiliencia materna; de forma específica, existió una correlación entre las dos dimensiones posi- tivas de la escala de funcionamiento familiar, lo que remite a que cuando los patrones de relación se caracterizan por satisfacción, intercambio de ideas y manifestaciones de afecto y respeto, existirá un mayor vínculo afectivo entre sus integrantes, aspectos que ante la presencia de discapacidad, representan mediadores positivos para la resiliencia materna.

A partir de la relación identificada entre la autodeterminación de la madre con las dimensiones que caracterizan al funcionamiento familiar, puede considerase a este como un mediador importante en el estudio de la resiliencia materna. En la presente investigación, se encontró que la autodeterminación se asoció con las cuatro dimensiones del funcionamiento familiar, lo que añade evidencias para sostener la importancia de analizar el ambiente familiar, en el estudio de la resiliencia materna ante la discapacidad intelectual. Ello coincide con lo señalado por Obrist et al. (2010), Spratt (2010) y Walsh (2003) cuando refieren que la familia, su funcionamiento positivo, así como la comunicación y la expresión adecuada del afecto, el poder llegar a acuerdos y la existencia de control adecuado (aspectos que remiten a la dimensión cohesión/reglas), determinan y pueden fomentar la resiliencia, aunque no lo documentan respecto a la madre en específico ni ante la discapacidad.

Esta asociación identificada entre la autodeterminación, tanto con el ambiente familiar positivo como con la cohesión/reglas, apoya lo señalado por Wehmeyer y Field (2007) y Spratt (2010) acerca de que algunas de las fortalezas de la familia en la que hay algún miembro con excepcionalidad, que promueven las características de resiliencia y contribuyen ante los cambios o crisis que se presentan, incluyen la adecuada comunicación, la involucración familiar, la satisfacción con la calidad de vida, las celebraciones, la fortaleza, el tiempo compartido, las rutinas y las tradiciones, cuestiones evaluadas en la dimensión ambiente familiar positivo.

También se identificó, en el presente estudio, que entre más autodeterminada sea la madre, menos existirá entre los miembros de su familia: 
a) falta de claridad en las reglas que rigen el comportamiento, límites confusos y dificultad para demostrar sentimientos, cuestiones relativas a la dimensión mando/problemas en la expresión de sentimientos y b) baja tolerancia, antagonismo y desvío de problemas, aspectos considerados en la hostilidad/evitación del conflicto. Ello refiere a los aspectos anotados por Spratt (2010) que no promueven características de resiliencia en el adulto que está a cargo del algún menor que presenta problemas en el desarrollo de habilidades sociales y educativas o que ha sido maltratado, es decir, existencia de un ambiente familiar caracterizado por deterioro en los vínculos de protección y apego, la expresión del afecto, la comunicación y los sentimientos de satisfacción.

La resiliencia contribuye al desarrollo socialmente aceptado de las madres y a que asuman un papel cada vez más activo ante el ambiente y la discapacidad intelectual de los hijos, por lo que se precisa realizar acciones de evaluación e investigación que favorezcan su identificación oportuna, máxime si habitan en un ambiente marginado como el del presente trabajo. A partir de esto, podrán elaborarse e instrumentarse programas de intervención que promuevan, de forma proactiva o reactiva, el desarrollo de características de resiliencia materna como la autodeterminación, así como los recursos que la favorecen, especialmente si se consideran los índices existentes respecto a discapacidad intelectual en México y la urgente necesidad de contribuir tanto a la inclusión y desarrollo de los hijos con discapacidad como a la corresponsabilidad escuela-familias.

A partir de esto, el presente trabajo, realizado desde un enfoque ecosistémico, aporta evidencia a la práctica y la investigación en la educación en general y, en particular, a la educación especial, pues a la fecha se ha otorgado poca atención a la interacción entre estas y la resiliencia materna (Gardynik \& McDonald, 2005; Gargiulo, 2012). Se precisa el conocimiento y entendimiento de las experiencias realizadas en contextos reales, pues estas poseen significado y representan el aprendizaje aprendido por quienes participan, lo que debe contribuir a la reflexión y guiar las acciones.

\section{Referencias}

Barudy, J. \& Dantagnan, M. (2005). Los buenos tratos en la infancia. Parentalidad, apego y resiliencia. Barcelona: Gedisa.

Breakwell, G. (2009). The psychology of risk. Cambridge: Cambridge University Press.

Farkas-Klein, C. (2008). Escala de evaluación parental (EEP): desarrollo, propiedades psicométricas y aplicaciones. Universitas Psychologica, 7(2), 457-467.

Flippin, M. \& Crais, E. (2011). The need for more effective father involvement early autism intervention. Journal of Early Intervention, 33(1), 24-50.

García-Méndez, M., Rivera, S., Reyes-Lagunes, I. \& Díaz-Loving, R. (2006). Construcción de una escala de funcionamiento familiar. Revista Iberoamericana de Diagnóstico y Evaluación Psicológica, 2(22), 91-110.

Gardynik, U. \& McDonald, L. (2005). Implications of risk and resilience in the life of the individual who is gifted/learning disabled. Roeper Review, 27(4), 206-214.

Gargiulo, R. M. (2012). Special education in contemporary society. An introduction to exceptionality. Belmont, CA: Wadsworth/Thomson Learning.

Henley, R. (2010). Resilience enhancing psychosocial programmes for youth in different cultural contexts. Progress in Development Studies, 10(4), 295-307.

Luthar, S. (2006). Resilience and development. En D. Cicchetti \& J. Cohen (Eds.), Developmental psychopathology (pp. 739-795). New York: Wiley.

Masten, A. (2007). Resilience in developing systems. Development and Psychopathology, 19(3), 921-930.

México, Gobierno Federal, Secretaría de Educación Pública. (2011). Modelo de atención de los Servicios de Educación Especial. México: Autor.

México, Instituto Nacional de Estadística, Geografía e Informática. (2010a). Demografía de la delegación Iztacalco. Disponible en http://www.iztacalco. df.gob.mx

México, Instituto Nacional de Estadística, Geografía e Informática. (2010b). Discapacidad en México. Disponible en http://cuentame.inegi.org.mx

Obrist, B., Pfeiffer, C. \& Henley, R. (2010). Multi-layered social resilience in context: A new approach in mi- 
tigation research. Progress in Development Studies, 10(4), 283-293.

Organización de las Naciones Unidas. (2006). Convención internacional sobre los derechos de las personas con discapacidad. París: Autor.

Pedraza-Vargas, S., Perdomo-Carvajal, M. \& Hernández, M. (2009). Terapia narrativa en la co-construcción de la experiencia y el afrontamiento familiar en torno a la impresión diagnóstica de TDAH. Universitas Psychologica, 8(1), 199-214.

Rodríguez, D. M., Alvarado, G. A. \& Moreno, F. M. (2007). Construcción participativa de un modelo socioecológico de inclusión social para personas en situación de discapacidad. Acta Colombiana de Psicología, 10(2), 181-187.

Roque, H. M. P., Acle, T. G. \& García, M. M. (2009). Escala de resiliencia materna: un estudio de validación en una muestra de madres con niños especiales. Revista Iberoamericana de Diagnóstico y Evaluación Psicológica, 1(27), 107-132.

Rutter, M. (1987). Psychosocial resilience and protective mechanisms. American Journal Orthopsychiatry, 57(3), 316-329.
Spratt, T. (2010). Families with multiple problems: Some challenges in identifying and providing services to those experiencing adversities across the life course. Journal of Social Work, 11(4), 343-357. doi: 10.1177/1468017310379256

Waller, M. (2001). Resilience in ecosystemic context: Evolution of the concept. American Journal of Orthopsychiatry, 71(3), 290-297.

Walsh, F. (2003). Family resilience. Strengths forged through adversity. En F. Walsh (Ed.), Normal family processes (pp. 399-423). New York: Guilford.

Wehmeyer, M. \& Field, S. (2007). Self-determination. Thousand Oaks, CA: Corwin Press.

Werner, E. E. \& Smith, R. S. (1992). Overcoming the odds. Ithaca, New York: Cornell University Press.

Whitley, R. (2012). Religious competence as cultural competence. Transcultural Psychiatry, 49(2), 245260. doi: $10.1177 / 1363461512439088$

World Health Organization. (2011). Disabilities. Disponible en http://www.who.int 